\title{
Systematic approach to CPR training urged
}

$\mathrm{C}$ anada should develop a systematic and compulsory approach to cardiopulmonary resuscitation (CPR) and automated external defibrillator training for all high school students and seniors, experts say.

There's a particular need to train more people aged 55 or older as they are most likely to make use of CPR training, given that $85 \%$ of cardiac arrests occur in the home in the presence of family, argues Dr. Christian Vaillancourt, research chair in emergency cardiac resuscitation at the University of Ottawa in Ontario and principle author of a Canadian Association of Emergency Physicians (CAEP) proposal to introduce compulsory CPR training as a condition of graduation from Canadian high schools.

An estimated 45000 Canadians suffer cardiac arrests annually and less than $5 \%$ of those who suffer cardiac arrests outside of a hospital survive, according to the Heart and Stroke Foundation of Canada.

"It must become a moral obligation and a social expectation that bystanders will perform CPR when they witness a cardiac arrest," states the CAEP position statement on bystander cardiopulmonary resuscitation (http://caep.ca/sites /default/files/caep/files/cjem-position -bystander-cr.pdf). "The general population must come to understand that cardiac resuscitation is much more likely to be successful when CPR is started promptly, and the victims of cardiac arrest will almost certainly die if lay witnesses do not intervene. There must be widespread recognition that CPR is a simple but vital life skill everyone should learn and then put into practice in emergency situations."

Along with mandatory CPR training in schools, the position paper urges tax exemptions for CPR education. "Many people, including low income senior citizens, choose not to enroll in a CPR course due to the expense. A $100 \%$ tax rebate would clearly offset this obstacle. More importantly, it would provide

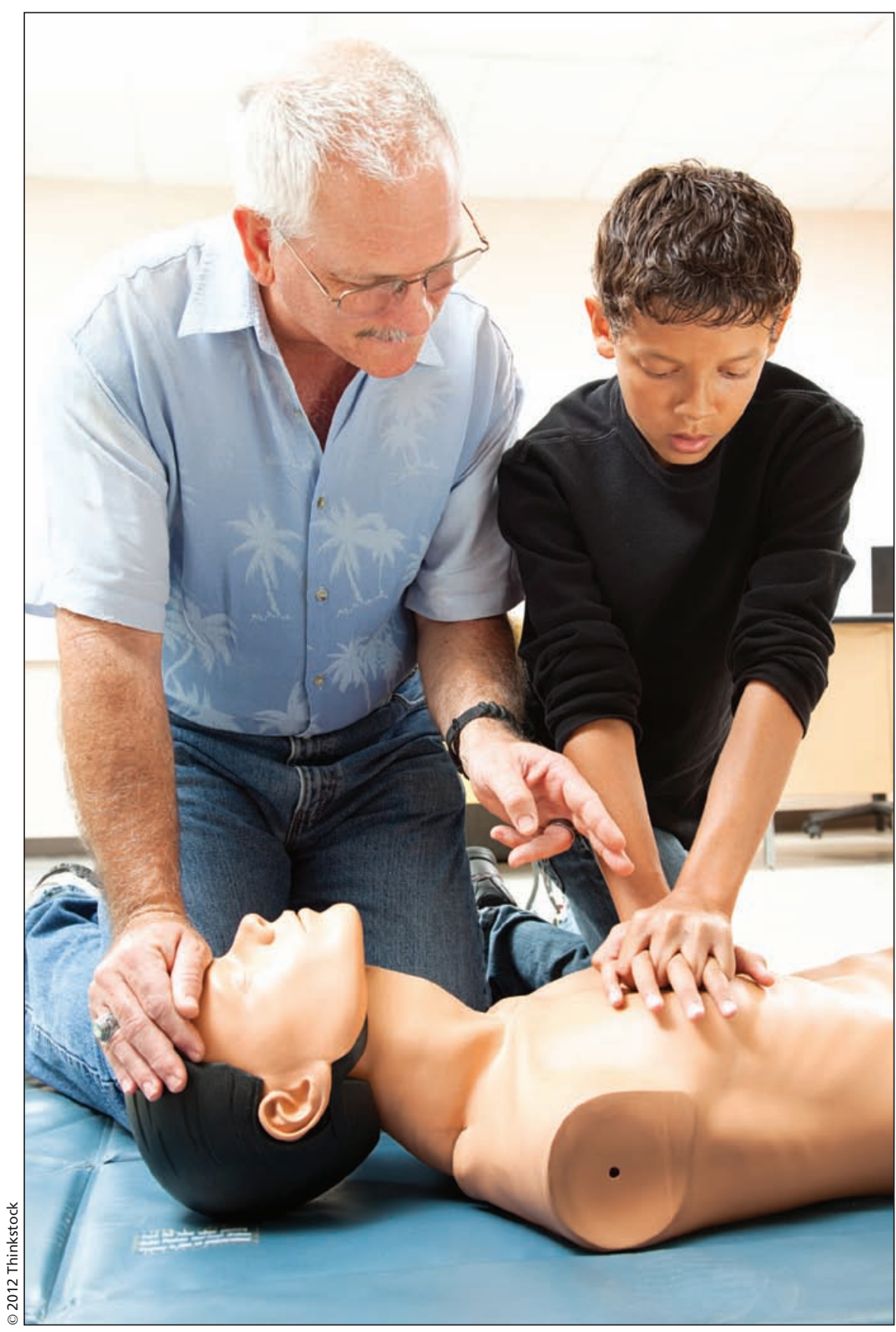

As an estimated $85 \%$ of cardiac arrests occur in the home in the presence of family, experts say it's particularly critical to provide CPR training for the elderly and the young, as they're most likely to find themselves in situations where they can provide assistance.

a compelling tax incentive to a large company to sponsor CPR education for its employees which, in turn, would ensure a safer workplace."
The CAEP, which believes all Canadians should be trained in CPR, notes that an estimated $60 \%$ of all Canadians have received some measure of such training 
in their lifetimes but that those skills "are often not practiced and kept up-to-date, leading to hesitation and inaction when faced with a cardiac arrest situation."

Vaillancourt says that compulsory CPR training for high school students would be "a step in the right direction" but it's even more critical to target training at older adults, as they're at highest risk of heart attacks. "If you look at the immediate impact of teaching high school kids CPR, you would not change bystander CPR rates tomorrow in that these kids are not in direct contact with the most likely victims."

"Our real target population would be the spouse of the 69-year-old man," notes Vaillancourt. "This is investing in the future. And really if we want to change numbers now we have to concentrate as well on senior citizens."

But there's still a need to train students as well, notes Dr. Justin Maloney, an emergency physician at the Ottawa Hospital and medical director of the Advanced Coronary Treatment (ACT) Foundation, a national charity organization based in Ottawa that is "dedicated to establishing free CPR as a mandatory program in every Canadian high school."

"I don't think we're going to create a generation of students who are going to be the actual witnesses to a lot of the people that are having cardiac arrests," Maloney says. "When we talk about CPR for kids, this is a long-term investment and culture change."
But it's not just about teaching kids the "thump and blow of CPR," Maloney adds. "The idea is not to just have a student know everything about CPR and walk away as a competent CPR citizen provider, but also to instill an attitude within that student that says he's going to do something in the face of an emergency."

Students are uniquely suited to adopting CPR as a life skill, says Sandra Clarke, executive director of the ACT Foundation. "Many students, when we interview them after they've saved a life, they say 'I was just doing what miss or sir told me I was supposed to do.' They're so modest, but by learning it in school, it becomes something very natural for them to do." The program also ensures "every young person brings these skills to not only their present but future families and communities."

Created in 1985, the ACT Foundation provides equipment, such as training mannequins, free to schools that make CPR training a component of their curriculum, typically for physical education classes.

The foundation enlists local groups such as St. John Ambulance Canada and the Heart and Stroke Foundation of Canada to offer a one-day workshop that will train teachers as CPR instructors. The only associated fees are the cost of a refresher course for teachers every three years, the cost of training new hires, and the payment of supply teachers. In return, participating school boards or schools must agree to include CPR training in their curriculum.

Schools typically opt to include such training in their physical education courses in grade 9 or 10, says Clarke, adding that the foundation is in the process of rolling out automated external defibrillator training to participating schools.

The foundation says that it has reached about 1600 schools, and trained about 1.8 million youth to date. CPR training is included in provincial secondary school curricula for one grade level in Ontario, Alberta and Manitoba, and is a component in the curricula of the majority of high schools in Quebec and British Columbia, one school in Nunavut and a handful of other schools in the remaining provinces, according to the foundation.

In the United States, Alabama, Indiana, Louisiana, North Carolina, Nevada, Pennsylvania, Rhode Island and Texas, as well as the District of Columbia, have made CPR and automated external defibrillator training a condition of high school graduation, as advocated by the American Heart Association (http://newsroom.heart.org/pr /aha/1220.aspx?link_page_rss=213053). Several other states have introduced legislation this year to follow suit. Michael Monette, CMAJ

CMAJ 2012. DOI:10.1503/cmaj.109-4278 